\title{
PEAKONS, R-MATRIX AND TODA LATTICE
}

\author{
O.Ragnisco ${ }^{(a)}, M \cdot$ Bruschi $^{(b)}$ \\ Istituto Nazionale di Fisica Nucleare, Sezione di Roma, P.le A.Moro 2, 00185 Roma,Italia \\ (a) Dipartimento di Fisica E.Amaldi, III Università di Roma \\ (b) Dipartimento di Fisica, Università di Roma "La Sapienza"
}

\begin{abstract}
The integrability of a family of hamiltonian systems, describing in a particular case the motion of N "peakons" (special solutions of the so-called Camassa-Holm equation) is established in the framework of the $r$-matrix approach, starting from its Lax representation. In the general case, the $r$-matrix is a dynamical one and has an interesting though complicated structure. However, for a particular choice of the relevant parameters in the hamiltonian (the one corresponding to the pure "peakons" case), the $r$-matrix becomes essentially constant, and reduces to the one pertaining to the finite (non-periodic) Toda lattice. Intriguing consequences of such property are discussed and an integrable time discretisation is derived.
\end{abstract}




\section{Introduction}

Interest in integrable Nonlinear Partial Differential Equations (NLPDEs) modelling physical phenomena and possessing "exhotic" localized solutions has been revived in recent years thanks to the work of Camassa and Holm [1].

Namely, in [1] an integrable NLPDE, included as a special case in the general scheme provided by Fokas and Fuchssteiner in 1981 [2], has been rederived in the context of water waves, and it has been shown to possess, under suitable assumptions, a new kind of localized solutions, denoted as "peakons".

On the other hand, a fruitful research activity has been in the past devoted to describe the time behaviour of the variables characterizing classes of special solutions of integrable NLPDEs in terms of integrable hamiltonian systems with finitely many degrees of freedom [3 - 7]. Quite naturally then, a similar approach has been pursued to investigate the N"peakons" dynamics, by different authors with different perspectives, and results have been

remarkable $[1,8,9]$. In particular, in ref.[9] it has been proven the complete integrability of the hamiltonian system defined, in canonical coordinates, by the following Hamilton's function:

$$
\mathcal{H}=\frac{1}{2} \sum_{j=1}^{N} \sum_{k=1}^{N} p_{j} p_{k}\left(\lambda+\mu \cosh \left[\nu\left(q_{j}-q_{k}\right)\right]+\mu^{\prime} \sinh \left[\nu\left|q_{j}-q_{k}\right|\right]\right)
$$

which reduces, in the special case $\mu^{2}=\left(\mu^{\prime}\right)^{2}$, to the one describing the motion of $\mathrm{N}$ "peakons" of the Camassa-Holm equation.

In this paper, the complete integrability of the hamiltonian (1.1) will be proven in the framework the classical $r$-matrix approach $[10,11]$. In this way, we will not only succeed in casting the system under scrutiny in a general unifying scheme, but we will also be able to uncover the basic features that render the pure "Peakons-Lattice" case $\mu^{2}=\left(\mu^{\prime}\right)^{2}$ (hereby denoted as PL) quite peculiar with respect to the general case.

In Section 2, starting form the Lax representation constructed in [8], we derive the $r$ matrix for the general case (that will be in the following denoted as GL), which turns out to be of dynamical type; its elegant structure allows one to give a neat characterisation of the Lax pair associated with any of the flows commuting with the $\mathcal{H}$ flow. In Section 3 , we restrict our considerations to PL, and observe first of all that its $r$-matrix is essentially constant, and is identical with the one pertaining to the finite Toda lattice; the two systems are thus endowed with the same Lie-Poisson structure. Guided by the above finding, we prove that the PL dynamics is just, in different coordinates, one of the compatible dynamics of the Toda lattice. Moreover, by using the factorisation (QR) algorithm [12], we construct an integrable time discretisation (Backlund transformation) for PL. Some possible extensions 
are outlined in Section 4.

\section{The $r$-matrix for the General Lattice}

First of all. we write down explicitly the Hamilton's equations associated with (1.1):

$$
\begin{gathered}
\dot{p}_{j}=-\frac{\partial \mathcal{H}}{\partial q_{j}}=p_{j} \sum_{k} p_{k}\left\{-\mu \sinh \left[\nu\left(q_{j}-q_{k}\right)\right]+\mu^{\prime} \operatorname{sign}\left(q_{j}-q_{k}\right) \cosh \left[\nu\left(q_{j}-q_{k}\right)\right]\right\} \\
\dot{q}_{j}=\frac{\partial \mathcal{H}}{\partial p_{j}}=\sum_{k} p_{k}\left\{\lambda+\mu \cosh \left[\nu\left(q_{j}-q_{k}\right)\right]+\mu^{\prime} \sinh \left[\nu\left|q_{j}-q_{k}\right|\right]\right\}
\end{gathered}
$$

We shall assume both $\mu$ and $\mu^{\prime}$ not equal to 0 . Indeed, the behaviour of the system in these special cases has been already investigated by F.Calogero in a previous paper [13], and

consequently will not be considered here. With no restriction, we can then rescale to 1 one of the parameters, say $\mu$, so that PL corresponds to $\mu^{\prime}= \pm 1$.

Following basically the notations used in [8], we write the Lax representation for (2.1) in the form:

$$
\dot{L}=[L, M]
$$

where $L, M$ are $N \times N$ matrices, whose entries read:

$$
\begin{gathered}
L_{j k}=\sqrt{p_{j} p_{k}} \alpha\left(q_{j}-q_{k}\right) \\
M_{j k}=-2 \kappa \sqrt{p_{j} p_{k}} \alpha^{\prime}\left(q_{j}-q_{k}\right)
\end{gathered}
$$

In $(2.2 \mathrm{c})$ a prime denoted differentiation with respect to the argument, and the function $\alpha(x)$ is given by:

$$
\alpha(x)=\cosh \frac{\nu x}{2}+\rho \sinh \frac{\nu|x|}{2}
$$

with:

$$
\mu^{\prime}=-\frac{2 \rho}{1+\rho^{2}} ; \kappa=\frac{2}{1+\rho^{2}}
$$

The hamiltonian (1.1) is then the following simple function of the invariants of the matrix $L$ :

$$
\mathcal{H}=-\kappa \operatorname{tr} L^{2}+(\lambda+\kappa+1)(\operatorname{tr} L)^{2}
$$


As the term $(\operatorname{tr} L)^{2}$ merely generates on overall translation of the system, one can assume $\lambda+\kappa+1=0$ with no essential lost of generality.

If we rewrite for a moment the Hamiltonian (1.1) in the form:

$$
\mathcal{H}=\sum_{j, k} p_{j} p_{k} f\left(q_{j}-q_{k}\right)
$$

with

$$
f(x)=\lambda+1+\kappa\left[1-\alpha^{2}(x)\right]
$$

we readily discover two important features of the dynamics, which merely follow from the even nature of the function $f(x)$, namely:

i) The $N$ coordinates $q_{j}$ keep their own initial ordering (see also [1]), as we have:

$$
\frac{\partial}{\partial t} \operatorname{sign}\left(q_{j}-q_{k}\right)=0
$$

Hence, we can assume "natural ordering": $q_{1} \leq q_{2} \leq \cdots q_{N}$. Physically, we would say that the particles do not cross each other.

ii) The conjugated momenta $p_{j}$ keep their own initial sign, as we have:

$$
\frac{\partial}{\partial t} \operatorname{sign} p_{j}=0
$$

In particular, there are no "dynamical ambiguities" in the expression $\sqrt{p_{j} p_{k}}$ occurring in formulas (2.2).

We further observe that Newton equations can be readily derived for the flow generated by (1.1). The most instructive procedure is to introduce a Lagrangian through a Legendre transformation:

$$
\mathcal{L}(q, \dot{q})=-\mathcal{H}(p, q)+\sum_{k} p_{k} \dot{q}_{k}=\mathcal{H}(p, q)=\frac{1}{2} \sum_{j k} \dot{q}_{j} \dot{q}_{k} G_{j k}(q)
$$

$G(q)$ being the inverse of the matrix $(F(q))_{j k} \equiv f\left(q_{j}-q_{k}\right)$. From (2.5) is natural to interpret the dynamics under scrutiny as a geodesic motion on a manifold characterized by the metric tensor $G(q)[1]$.

We now turn to the evaluation of the $r$-matrix, which is well known to be a basic tool in the theory of integrable systems $[10,11]$. As it has been shown for the first time in [11], whenever a hamiltonian system is associated with a Lax matrix whose eigenvalues - assumed to be simple, at least locally - are in involution for a given Poisson bracket, it enjoys an $r$-matrix representation of the form: 


$$
\left\{L_{1}, L_{2}\right\}=\left[r_{12}, L_{1}\right]-\left[r_{21}, L_{2}\right]
$$

In (2.6) we have used the standard notations:

$$
\begin{gathered}
L_{1} \equiv L \otimes \mathbf{1}=\sum_{i} L_{i} e^{i} \otimes \mathbf{1} \\
L_{2} \equiv \mathbf{1} \otimes L=\sum_{i} L_{i} \mathbf{1} \otimes e^{i} \\
r_{12}=\sum_{i, k} r_{i k} e^{i} \otimes e^{k} \quad r_{21}=\sum_{i, k} r_{k i} e^{i} \otimes e^{k}
\end{gathered}
$$

where $\left\{e^{i}\right\}_{i=1}^{M}$ is a basis for the matrix Lie-algebra $\mathcal{G}$ which $L$ belongs to, and, in general, the coefficients $r_{i k}$ will be functions on the phase space (dynamical r-matrix).

Conversely, whenever an element $r \in \mathcal{G} \otimes \mathcal{G}$ exists such that formula (2.6) holds, the eigenvalues of $L$ and in general its invariant functions are in involution.

We recall that the trace form on $\mathcal{G}$ allows one to identify $\mathcal{G}$ with its dual (the space of linear functions on $\mathcal{G}$ ), and to consider the $r$-matrix as an endomorphism $R$ on $\mathcal{G}$, rather than as an element of $\mathcal{G} \otimes \mathcal{G}$, so that in such a "dual" picture, eq.(2.6) induces the following Lie-Poisson bracket between two functions on $\mathcal{G}$ :

$$
\{f, g\}_{L}=\left(L,[d f, d g]_{R}\right)
$$

with

$$
[X, Y]_{R}=[X, R(Y)]+[R(X), Y] \quad(X, Y \in \mathcal{G})
$$

and:

$$
R(X)=\sum_{j, k} r_{j k} e^{(i)}\left(e^{(k)}, X\right)
$$

In our case, everything is very simple. The algebra $\mathcal{G}$ is just $g l(N, \mathbf{R})$, so that we work with the standard (Weyl) basis :

$$
\left(E_{j k}\right)_{l m}=\delta_{j l} \delta_{k m}
$$

and we write:

$$
\left\{L_{1}, L_{2}\right\}=\sum_{j k ; l m}\left\{L_{j k}, L_{l m}\right\} E_{j k} \otimes E_{l m}
$$




$$
r_{12}=\sum_{j k ; l m} r_{j k ; l m} E_{j k} \otimes E_{l m}
$$

The basic ingredient in the evaluation of the $r$-matrix is the fundamental Poisson bracket $\left\{L_{j k}, L_{l m}\right\}$, whose expression in our case reads:

$$
\begin{gathered}
\left\{L_{j k}, L_{l m}\right\}=\frac{1}{4}\left[\delta_{j l} \sqrt{p_{k} p_{m}}\left(\alpha_{m j} \alpha_{j k}\right)^{\prime}+\delta_{j m} \sqrt{p_{k} p_{l}}\left(\alpha_{l j} \alpha_{j k}\right)^{\prime}\right. \\
\left.-\delta_{k l} \sqrt{p_{j} p_{m}}\left(\alpha_{m k} \alpha_{k j}\right)^{\prime}-\delta_{k m} \sqrt{p_{j} p_{l}}\left(\alpha_{l k} \alpha_{k j}\right)^{\prime}\right]
\end{gathered}
$$

In formula (2.9), a prime denotes again differentiation, and we have used the shorthand notation $\alpha_{j k} \equiv \alpha\left(q_{j}-q_{k}\right)$; in the derivation of (2.9) a crucial role is played by the formula:

$$
\left(\frac{\partial}{\partial x}+\frac{\partial}{\partial y}\right) \alpha(x) \alpha(y)=\frac{\nu}{4}\left[\left(1-\left(\mu^{\prime}\right)^{2}\right) \sinh [\nu(x+y)]-2 \mu^{\prime} \alpha(x+y)(\operatorname{sign} x+\operatorname{sign} y)\right]
$$

Looking carefully at (2.10) we readily understand that there are two essentially different cases, the PL case $\left(\rho^{2}=1 \rightarrow\left(\mu^{\prime}\right)^{2}=1\right)$ and the GL case $\left(\rho^{2} \neq 1\right)$.

A comparison of formulas (2.6) and (2.9) yields the following expression for the $r$-matrix:

$$
\begin{gathered}
r_{12}=-\frac{\nu}{8} \rho \sum_{j k} \operatorname{sign}\left(q_{j}-q_{k}\right) E_{j k} \otimes\left(E_{j k}+E_{k j}\right) \\
+\frac{\nu}{4}\left(1-\rho^{2}\right) \sum_{j k l} S_{k l}\left(E_{j k}-E_{k j}\right) \otimes\left(E_{j l}+E_{l j}\right),
\end{gathered}
$$

where the symmetric matrix $S$ is determined, up to functions of $L$, by the linear system:

$$
[S, L]_{j k}=\sinh \left[\frac{\nu}{2}\left(q_{j}-q_{k}\right)\right]:=A_{j k}
$$

It is instructive to consider the dual picture as well, where the endomorphism $R(X)(2.7 \mathrm{c})$ reads:

$$
R(X)=\frac{\nu}{4}\left(\rho\left[\left(X^{(s)}\right)_{-}-\left(X^{(s)}\right)_{+}\right]+2\left(1-\rho^{2}\right)\left[S, X^{(s)}\right]\right) .
$$

In $(2.13)$ by $X^{(s)}$ we have denoted the symmetric part of the matrix $X$, and the suffix $+(-)$ stands for the strictly upper (strictly lower) triangular part. To write down (2.13) we took advantage of the natural ordering, replacing $\operatorname{sign}\left(q_{j}-q_{k}\right)$ by $\operatorname{sign}(j-k)$.

It is now a straightforward task to write down the Lax representation for the commuting hamiltonian flows generated by the invariant functions $\mathcal{F}^{(k)} \equiv \frac{1}{k+1} \operatorname{tr} L^{k+1}$. In fact we have:

$$
\frac{\partial L}{\partial t_{k}} \equiv\left\{\mathcal{F}^{(k)}, L\right\}=\left[L, M^{(k)}\right]
$$


with:

$$
M^{(k)}=R\left(L^{k}\right)=\frac{\nu}{8}\left[\rho\left(\left(L^{k}\right)_{-}-\left(L^{k}\right)_{+}\right)+\left(\rho^{2}-1\right) \sum_{j=0}^{k-1} L^{j} A L^{k-1-j}\right.
$$

Note that a remarkable simplification in (2.14a) can be achieved thanks to the identity

$$
\left[L, \sum_{j=0}^{k-1} L^{j} A L^{k-1-j}\right]=\left[L^{k}, A\right]
$$

\section{The PL case and its connection with the Toda lattice}

As we have already noticed, the PL case corresponds to the special choice $\rho^{2}=1$, and is thus endowed with the $r$-matrix:

$$
r_{12}=-\frac{\nu}{8} \rho \sum_{j k} \operatorname{sign}\left(q_{j}-q_{k}\right) E_{j k} \otimes\left(E_{j k}+E_{k j}\right)
$$

or, in the dual picture:

$$
R_{P L}(X)=\frac{\nu}{4}\left(\rho\left[\left(X^{(s)}\right)_{-}-\left(X^{(s)}\right)_{+}\right]\right.
$$

which is just one of the $r$-matrices that can be associated with the finite nonperiodic Toda lattice [14]. Such striking coincidence strongly suggests to give a closer look to the PL case, to uncover possible further connections with the Toda system.

To this aim, we first notice that the inverse of the $L$ matrix $(2.2 \mathrm{~b})$ is in this case easily computable.

Clearly, the problem reduces to the evaluation of the inverse of the matrix

$$
C_{j k} \equiv \alpha_{j k}=\exp \left( \pm \frac{\nu}{2}\left|q_{j}-q_{k}\right|\right)
$$

If we introduce the quantities:

$$
e_{j} \equiv \exp \left( \pm \frac{\nu}{2}\left(q_{j+1}-q_{j}\right)\right.
$$

and take into account the natural ordering, we readily see that:

$$
C_{j, j+r}=e_{j} e_{j+1} \ldots e_{j+r-1}
$$

so that the matrix $C$ can be explicitly inverted yielding a tridiagonal Jacobi matrix. The final result is the following: 


$$
L^{-1}=\left(\begin{array}{cccc}
B_{1} & A_{1} & 0 & \ldots \\
A_{1} & B_{2} & A_{2} & \ldots \\
0 & \ddots & \ddots & \ddots \\
\vdots & \ldots & A_{N-1} & B_{N}
\end{array}\right)
$$

where:

$$
A_{j}=-\frac{1}{p_{j} p_{j+1}} \frac{e_{j}}{1-e_{j}^{2}} ; \quad B_{j}=\frac{1}{p_{j}} \frac{1-e_{j-1}^{2} e_{j}^{2}}{\left(1-e_{j-1}^{2}\right)\left(1-e_{j}^{2}\right)}
$$

with $e_{0}=e_{N}=0$.

The isospectral flows of $L^{-1}$ are of course characterized by the dynamical equations:

$$
\frac{\partial L^{-1}}{\partial t_{k}}=\left[L^{-1}, R_{P L}\left(L^{k}\right)\right]
$$

and in particular we have:

$$
\left\{\mathcal{H}, L^{-1}\right\}=\left[L^{-1}, R_{P L}(L)\right]
$$

The above equation can be trivially but usefully written in terms of the new dynamical variable $\Lambda \equiv L^{-1}$, taking the form:

$$
\dot{\Lambda}=\left[\Lambda, R_{P L}\left(\Lambda^{-1}\right)\right]=\left[\Lambda, R_{P L}\left(\nabla_{\Lambda} \ln \operatorname{det} \Lambda\right)\right]
$$

Thus we have the following Proposition:

"When the variables $(q, p)$ evolve according to the Hamiltonian (1.1), the "Flaschka variables" $(A, B)$, defined through $(3.3,3.1 \mathrm{~b})$ evolve according to the Hamiltonian $\ln \operatorname{det} \Lambda$ ". In this sense, the PL lattice is just one of the commuting flows of the (finite, nonperiodic) Toda hierarchy.

Encouraged by the above property, we have further exploited the connection with the Toda system to derive an integrable discretisation of the PL system, i.e. a symplectic map preserving the conserved quantities of the continuous-time model. Like in the Toda system, the basic tool is the factorisation of the Lax matrix [12, 15]. Taking care of the natural ordering, and assuming for the sake of simplicity the $+\operatorname{sign}$ in $(3.1 a, b)$ it can be written as:

$$
L=\sum_{j} p_{j} E_{j j}+\sum_{k>j} \sqrt{p_{j} p_{k}} \exp \left[\frac{\nu}{2}\left(q_{k}-q_{j}\right)\right] E_{j k}+\sum_{k<j} \sqrt{p_{j} p_{k}} \exp \left[\frac{\nu}{2}\left(q_{j}-q_{k}\right)\right] E_{j k}
$$

It is then possible to find out explicitly (and uniquely, up to signs) an upper triangular matrix $\mathcal{U}(L)$ : 


$$
\mathcal{U}(L) \equiv \sum_{j} \sum_{k \geq j} u_{j k} E_{j k}
$$

such that:

$$
L=\mathcal{U}(L) \times[\mathcal{U}(L)]^{t}
$$

where the superscript $t$ denotes transposition. In fact one finds:

$$
u_{j k}^{2}=p_{j}\left[\exp \left[\nu\left(q_{k}-q_{j}\right)\right]-\exp \left[\nu\left(q_{k+1}-q_{j}\right)\right]\right.
$$

The above expression holds for $1 \leq k \leq N$, provided we set $q_{N+1}=-\infty$.

The new (or "Miura transformed") $L$, say $\tilde{L}$, is then given by:

$$
\tilde{L}=[\mathcal{U}(L)]^{t} \times \mathcal{U}(L)
$$

which yields for the entries $u_{j k}(3.6 \mathrm{a})$ the alternative expression:

$$
u_{j k}^{2}=\tilde{p}_{k}\left[\exp \left[\nu\left(\tilde{q}_{k}-\tilde{q}_{j}\right)\right]-\exp \left[\nu\left(\tilde{q}_{k}-\tilde{q}_{j-1}\right)\right]\right.
$$

Identifiying (3.6c) and (3.7b) we get, after some trivial manipulation:

$$
\frac{\tilde{p}_{k}}{\exp \left[\nu\left(q_{k}-\tilde{q}_{k}\right)\right]-\exp \left[\nu\left(q_{k+1}-\tilde{q}_{k}\right)\right]}=\frac{p_{j}}{\exp \left[\nu\left(q_{j}-\tilde{q}_{j}\right)\right]-\exp \left[\nu\left(q_{j}-\tilde{q}_{j-1}\right)\right]}
$$

The r.h.s. and the 1.h.s. of (3.8) must then be independent of the label, i.e. they have to be equal to the same constant, say $\beta$. Therefore, eq.(3.8) is in fact equivalent to the canonical transformation:

$$
\begin{aligned}
& \tilde{p}_{k}=\beta\left(\exp \left[\nu\left(q_{k}-\tilde{q}_{k}\right)\right]-\exp \left[\nu\left(q_{k+1}-\tilde{q}_{k}\right)\right]\right) \\
& p_{k}=\beta\left(\exp \left[\nu\left(q_{k}-\tilde{q}_{k}\right)\right]-\exp \left[\nu\left(q_{k}-\tilde{q}_{k-1}\right)\right]\right.
\end{aligned}
$$

whose generating function is:

$$
S(q, \tilde{q})=\frac{\beta}{2} \sum_{k}\left(\exp \left[\nu\left(q_{k}-\tilde{q}_{k}\right)\right]-\exp \left[\nu\left(q_{k+1}-\tilde{q}_{k}\right)\right]\right.
$$

The canonical transformation (3.9) can be interpreted as a discrete-time flow, by setting:

$$
\left(p_{j}, q_{j}\right)=\left(p_{j}(n), q_{j}(n)\right) ;\left(\tilde{p}_{j}, \tilde{q}_{j}\right)=\left(p_{j}(n+1), q_{j}(n+1)\right)
$$


and written in the following discrete Newton form in terms of $q=q(n), \tilde{q}=q(n+1), \hat{q}=$ $q(n-1)$ :

$$
\exp \left[\nu\left(\hat{q}_{j}-q_{j}\right)\right]-\exp \left[\nu\left(\hat{q}_{j+1}-q_{j}\right)\right]=\exp \left[\nu\left(q_{j}-\tilde{q}_{j}\right)\right]-\exp \left[\nu\left(q_{j}-\tilde{q}_{j-1}\right)\right]
$$

Moreover, as in the Toda case, such discrete flow can be explicitly integrated. Namely, if we denote $L=L(p, q)=L_{n}, \tilde{L}=L(\tilde{p}, \tilde{q})=L_{n+1}$, we get [15]:

$$
L_{n}=\mathcal{U}\left(L_{0}^{n}\right) L_{0}\left[\mathcal{U}\left(L_{0}^{n}\right)\right]^{-1}
$$

From (3.11a), looking at $n$ as at a discrete time, we can read off the interpolating hamiltonian $\mathcal{F}(L)$ defined through:

$$
L^{n}=\exp \left[n \nabla_{L} \mathcal{F}\right]
$$

whence it follows:

$$
\mathcal{F}(L)=\operatorname{tr} L(\ln L-I)
$$

In connection with the above result, it might be worthwhile to recall that interest in integrable time-discretisation has been recently revived by a number of stimulating papers [16].

\section{Concluding remarks}

We would like to summarize here the main results derived in the present paper.

First of all, we have found the proper $r$-matrix formulation for the family of hamiltonian systems recently investigated in [8], containing as a special case the "peakons" system: we have provided an alternative proof of complete integrability and have exhibited a Lax representation for the various commuting flows. Secondly, we have noticed that the PL system enjoys the same $r$-matrix as the finite nonperiodic Toda system: this property led us to identify the PL system with one of the commuting flows of the Toda hierarchy and paved the way for the construction of an integrable time-discretisation.

Several questions are still open. The most natural one refers perhaps to the algebraic nature of the hamiltonian system corresponding to the special choice $\rho=0$ in (2.3) (yielding $\mu^{\prime}=0$ in $\left.(1.1)\right)$, already shown to be super-integrable in [13]: namely, is the " $r$-matrix" pertaining to this special case still a "good" one? We did not check Jacobi identity in this special case, but anyway observe that the corresponding Lax matrix is of rank 2, and thus one of the conditions which guarantee complete integrability is not fulfilled (in fact, 
complete integrability has been proven in [13] in a different way, not relying upon the Lax representation). A further open problem is the construction of an integrable discretisation for the whole family of hamiltonian systems, not only for the PL system: finding an explicit factorisation is however a rather hard task in the GL case, and so far we have not succeded. Finally, the existence of a bi-hamiltonian formulation is something to be investigated: in this context, the well known results available for the Toda system may be exploited in the PL case, while again the GL system needs a separate investigation, as the dynamical nature of the $r$-matrix makes the results derived for instance in [14] not immediately applicable. 


\section{References}

[1] R.Camassa, D.D. Holm, Phys. rev. Lett. 71 (1993), 1661-1664;

R. Camassa, D.D. Holm, J.M. Hyman, Adv. Appl. Math. 31 (1994), 1-33.

[2] A.S. Fokas, B. Fuchssteiner, Physica D4 (1981), 47-66.

[3] H. Airault, H.P. McKean, J. Moser, Comm. Pure Appl. Math. 30 (1977), 95-148.

[4] I.M. Krichever, Funct. Anal. Appl. 12 (1978), 50-61.

I.M. Krichever, Funct. Anal. Appl. 14 (1980), 282-290.

[5] O.I. Bogoyavlenski, S.P. Novikov, Funct. Anal. Appl. 13 (1976), 6.

[6] E. Previato, "The Calogero-Moser-Krichever System and Elliptic Boussinesq Solitons", in Hamiltonian Systems, Transformation Groups and Spectral Transform Methods, J.Harnad and J.Marsden eds, CRM Momtreal 1990.

[7] M. Antonowicz, S. Rauch-Wojciechowski, Phys. Lett. 147A (1990), 455;

M. Antonowicz, S. Rauch-Woijciechowski, J. Phys. A: Math. Gen 24 (1992), 5043.

[8] F.Calogero, J.P. Francoise, "A Completely Integrable Hamiltonian System", J. Math. Phys. (submitted to).

[9] M. Alber, "New types of soliton solutions for nonlinear equations", talk delivered at the Conference on Nonlinear Coherent Structures in Physics and Biology, held in Edinburg, Scotland, July 10-14, 1995.

[10] M.A. Semenov-Tianshanski, Funct. Anal. Appl. 17 (1983), 259.

[11] O. Babelon, C.M.Viallet, Phys. Lett. B 237 (1990), 411-416.

[12] W.W Symes, Invent. Math. 59 (1980), 13-51.

[13] F. Calogero, Phys. Lett. A 201 (1995).

[14] W. Oevel, O. Ragnisco, Physica A 161, (1989), 181-220.

[15] Yu. B. Suris, "New Discretisation of the Toda Lattice", Preprint University of Bremen, Centre for Complex Systems and Visualization, 1995.

[16] F.Nijhoff, G.D. Pang, "Discrete-time Calogero-Moser model and lattice KP equation", in Symmetries and Integrability of Difference Equations, D. Levi, P. Winternitz ans L. Vinet eds., CRM Montreal 1995;

F.Nijhoff, O.Ragnisco, V.Kuzsnetsov, "Integrable time-discretization of the RujisenaarsSchneider model", Comm. Math. Phys. (to appear);

Yu.B. Suris, "A discrete-time relativistic Toda lattice", Preprint University of Bremen, Centre for Complex Systems and Visualization, 1995. 